\title{
How do liquids confined at the nanoscale influence adhesion?
}

\author{
C Yang ${ }^{1}$, U Tartaglino ${ }^{1,2}$ and B N J Persson ${ }^{1}$ \\ ${ }^{1}$ IFF, FZ-Jülich, 52425 Jülich, Germany \\ ${ }^{2}$ DEMOCRITOS National Simulation Center, Via Beirut 2, 34014 Trieste, Italy
}

Received 3 August 2006, in final form 31 October 2006

Published 27 November 2006

Online at stacks.iop.org/JPhysCM/18/11521

\begin{abstract}
Liquids play an important role in adhesion and sliding friction. They behave as lubricants in human bodies, especially in the joints. However, in many biological attachment systems they act like adhesives, e.g. facilitating insects to move on ceilings or vertical walls. Here we use molecular dynamics to study how liquids confined at the nanoscale influence the adhesion between solid bodies with smooth and rough surfaces. We show that a monolayer of liquid may strongly affect the adhesion.
\end{abstract}

(Some figures in this article are in colour only in the electronic version)

Knowing the behaviour of liquids confined to small volumes between contacting surfaces is essential for the understanding of a vast array of common problems in science, such as biological interactions [1-3], crack propagation [4], molecular tribology and adhesion [3, 5-8]. Ultrathin confined films can have two completely opposite effects. On one hand, the confined liquid may behave like a lubricant to reduce adhesion and friction. For instance, when bones meet at a joint, they need a liquid in between to prevent them scraping against each other. The liquid is called a synovial liquid [9], which is made mainly of water and water-based long-chain polymers. Many internal organs in humans and other animals are separated by thin lubricant films. Examples include the cerebrospinal liquid in the brain [10], the pleural liquid in lungs [11] and water-based liquid in eyes. On the other hand, in some applications liquids behave like adhesives, e.g. in some biological attachment systems. Thus, for example, insects can secrete a liquid to enhance adhesion in order to walk on ceilings or vertical walls [1]. Geckos can also walk on ceilings due to their hierarchical hair structure which makes their adhesion pads elastically soft on all relevant length scales [12]. Recently scientists found that water monolayer-films, which always occur on hydrophilic (water-loving) surfaces in the normal atmosphere, enhance gecko adhesion $[13,14]$ to hydrophilic surfaces.

The influence of thin liquid layers on the effective adhesion between solids is well known to most of us from our everyday experience, e.g., when separating two (slightly wet) microscopy cover glass surfaces, or two gauge blocks (steel blocks with very smooth surfaces). At the 
microscale or nanoscale the increased adhesion resulting from the formation of water capillary bridges is one of the most severe problems in the production of micro-electro-mechanical systems (MEMS) [15-17]. In general, wetting liquids tend to increase the adhesion between solids with surface roughness, but at present it is not known exactly for which liquid coverage the pull-off force is maximal $[6,18]$. There are also many unsolved questions related to the influence of liquids on adhesion in biological systems, e.g., how is it possible for a gecko to move on a vertical stone wall during rain, and how do cells (in liquids) adhere to solids walls?

In this study we show that small amounts of confined liquid between smooth and randomly rough surfaces can influence adhesion. Using liquids that interact weakly and strongly with the walls (hydrophobic [19] and hydrophilic walls), we demonstrate that sometimes the liquid acts like a lubricant, as in some biological systems, such as in the eyes, and sometimes it behaves like a glue, as the insects' secretions when they walk on ceilings or vertical walls.

We have used molecular dynamics calculations to study the influence of liquids confined at the nanoscale on adhesion. We have simulated an elastic block approaching a hard substrate covered with octane molecules. The atoms in the bottom layer of the block form a simple square lattice with lattice constant $a=2.6 \AA$. The lateral dimensions are $L_{x}=N_{x} a$ and $L_{y}=N_{y} a$. For the block, $N_{x}=N_{y}=48$. Periodic boundary conditions are applied in the $x y$ plane. The lateral size of the block is equal to that of the substrate, but we used a different lattice constant, $b \approx a / \phi$, where $\phi=(1+\sqrt{5}) / 2$ is the golden mean, in order to avoid the pinning due to a perfect match of the crystal structures. The thickness of the block, $142 \AA$, is comparable to the lateral length of the system. A recently developed multiscale molecular dynamics description [20] let us properly describe the elastic response of the block without having to simulate too many atoms in the bulk. The Young's modulus of the block is $E=100 \mathrm{GPa}$ and the Poisson ratio is $v=0.3$. We consider both atomically flat and rough substrates. The profile of the rough substrate is self-affine fractal with root-mean-square (rms) amplitude $3 \AA$ and fractal dimension $D_{\mathrm{f}}=2.2$; it is prepared following the recipe in $[20,21]$.

The liquid between the walls is octane $\mathrm{C}_{8} \mathrm{H}_{18}$. A monolayer of liquid on the flat substrate is composed of 409 octane molecules. The simulations are carried out at room temperature, $T=300 \mathrm{~K}$, well above the melting point of octane $T_{\mathrm{m}}=216 \mathrm{~K}$. The octane molecules are treated with the united atom model: every molecule comprises eight particles corresponding to the groups $\mathrm{CH}_{2}$ and $\mathrm{CH}_{3}$. The optimized potential for liquid simulation (OPLS) [22, 23] is employed. The atoms of the two walls interact through the Lennard-Jones potential [20], with parameters $\sigma=3.28 \AA$ and $\epsilon=40 \mathrm{meV}$. The interaction between the walls' atoms and the groups $\mathrm{CH}_{2}$ and $\mathrm{CH}_{3}$ of the octane molecules are also given by the Lennard-Jones potential with $\sigma=3.28 \AA$, but two different interaction energies are considered: strong adsorbate interaction with $\epsilon=40 \mathrm{meV}$, and weak adsorbate interaction with $\epsilon=5 \mathrm{meV}$.

In the simulations we first let the octane deposit on the substrate and thermalize, then we bring the elastic block into contact with the substrate, and finally we pull the block up slowly until the two surfaces separate. The overall preparation of the system before retraction requires a simulation of 4-6 ns. Approach and retraction are realized by imposing a fixed speed $v=1 \mathrm{~m} \mathrm{~s}^{-1}$ to the outer surface of the block, while the wall in contact with the substrate and the bulk of the block are free to deform elastically. The pulling speed is small enough not to alter the pull-off process. Indeed we performed simulations (not shown) to detect when the pulling speed becomes relevant: we observed that the pull-off force changes significantly only for speeds above $50 \mathrm{~m} \mathrm{~s}^{-1}$. We speculate that for larger amounts of liquids and for chains longer than octane (more cross-linked) the influence of speed can appear at smaller velocities. In the process of detachment the interaction between the lubricated walls is attractive, and the adhesive force reaches a maximum at a given distance, which depends on the amount of liquid, and then it decreases. Figure 1(a) shows the maximum pulling force per unit area (pull-off 

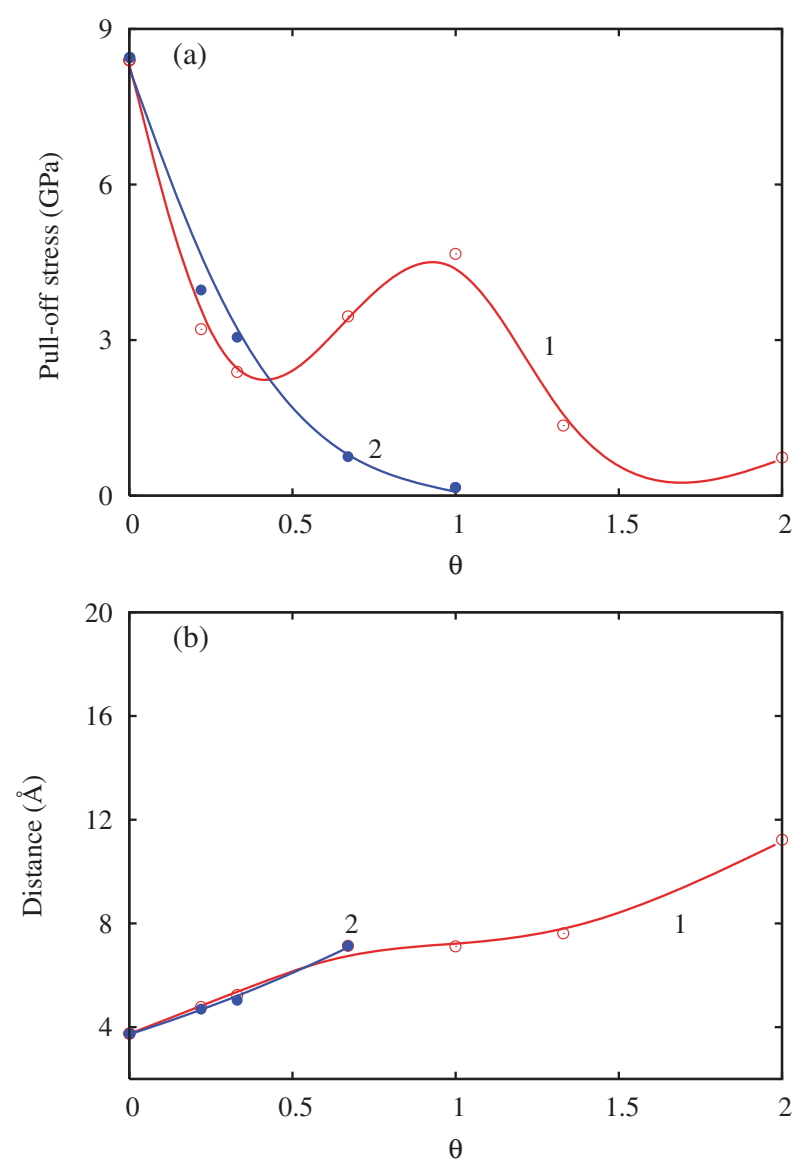

Figure 1. Pull-off on a flat substrate for strong adsorbate interaction (curve 1) and for weak adsorbate interaction (curve 2). (a) The maximum pull-off stress versus the liquid coverage $\theta$. (b) The separation between the walls where the stress is maximum versus $\theta$. Continuous lines: fit with smoothing cubic splines.

stress), as a function of liquid coverage $\theta$, for the case of two flat walls. The corresponding distance between the walls when the pulling force is maximum is plotted in figure 1(b).

We note that the direct interaction between the block and the substrate is negligible when the surfaces are separated by one adsorbate monolayer. The pull-off stress is maximum for clean and smooth surfaces since there is strong interaction between the wall atoms (LennardJones parameter $\epsilon=40 \mathrm{meV}$ ). The pull-off stress rapidly decreases with increasing amount of liquid between the surfaces. For $\theta<1$, the boundary line of an island of lubricant molecules can be considered as a crack edge, as shown in figure 2. During pull-off, high stress concentration occurs at the crack tip, and the interfacial crack propagation starts at the crack edge, resulting in the strong decrease in the pull-off force observed in the simulations for $\theta<1$.

Figure 2 shows the side view of the system before and after detachment when the coverage is $\theta=1 / 4$. For strong interaction between liquid and walls ((a) and (b)) not all molecules are trapped in one island. For weak adsorbate interaction ((c) and (d)) the liquid molecules experience smaller lateral energy barriers, and for $\theta<1$ they arrange themselves into a single 


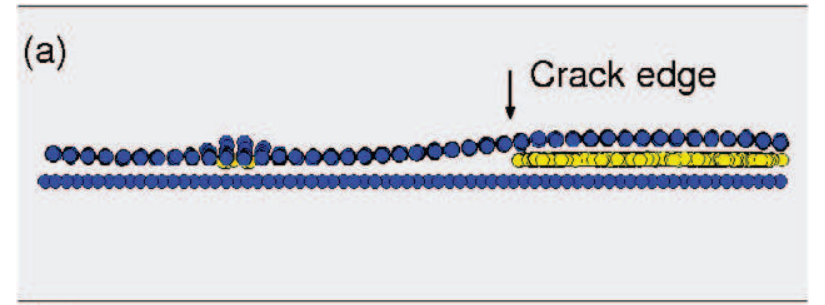

(b)
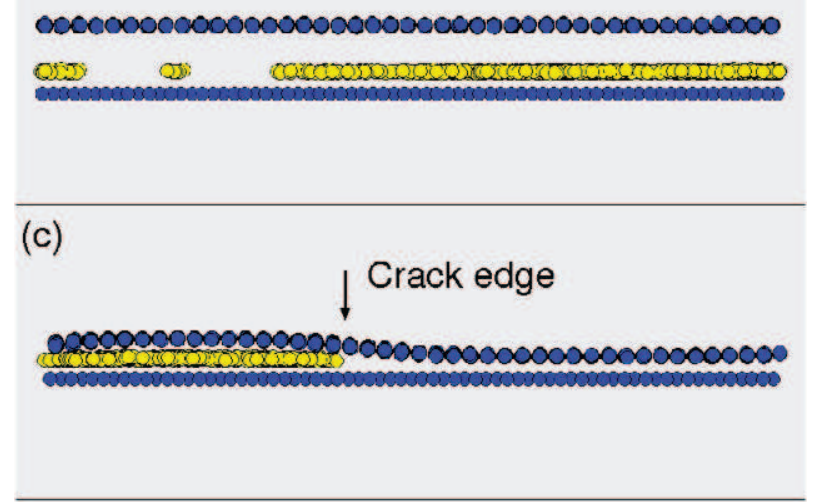

(d)

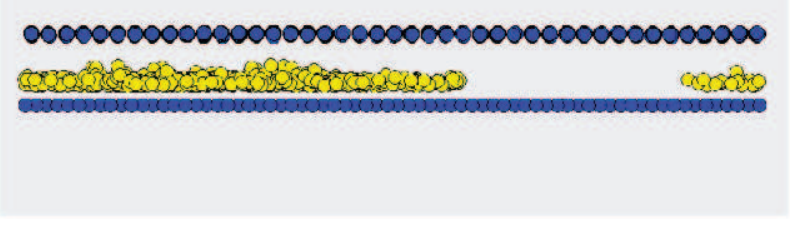

Figure 2. Side view of the system with coverage $\theta \approx 1 / 4$ during the pull-off. The light balls represent the $\mathrm{CH}_{2}$ and $\mathrm{CH}_{3}$ groups of the octane molecules. The dark balls are the atoms of the walls' surfaces. (a) Strong adsorbate interaction between the liquid and the walls; the separation happens by crack propagation. (b) After the detachment the block returns to its undeformed state. (c) and (d) during and after the detachment for the case of weak adsorbate interaction. The behaviour is the same as in the previous case.

large island, and thus less elastic energy is stored at the block-substrate interface in this case, resulting, at low liquid coverage, in a (slightly) stronger adhesion (or pull-off force) for weak adsorbate interaction as compared to the case of strong interaction. The morphology of the adsorbate depends on the preparation of the system. In an infinitely large system the liquid will always form finite clusters, whose size and density are determined by the competition between deposition and diffusion [24]. As a general rule the stronger interaction will give rise to smaller islands, so that for the same preparation the stronger adsorbate interaction will lead to a slightly lower adhesion, as shown by our simulations. Notice that after the detachment of the two walls all the liquid sticks to the substrate: indeed its adhesion energy to the substrate is larger because of the larger substrate atom density compared to that of the upper block.

For weak adsorbate interaction, the pull-off stress decreases monotonically with increasing $\mathrm{C}_{8} \mathrm{H}_{18}$ coverage. On the other hand, strong adhesive interaction causes an increase of the pulloff stress up to a maximum for the coverage $\theta=1$ corresponding to a complete monolayer. 

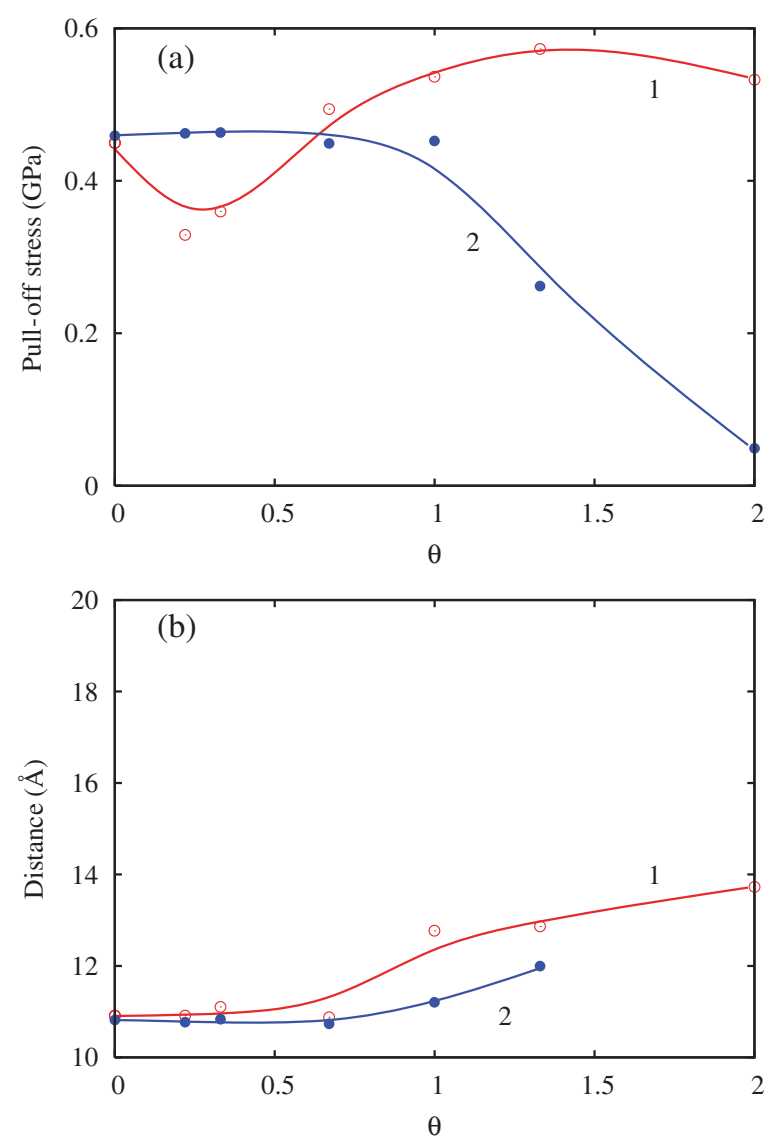

Figure 3. Pull-off on a rough substrate for strong adsorbate interaction (curve 1) and for weak adsorbate interaction (curve 2). (a) The maximum pull-off stress versus the liquid coverage $\theta$. (b) The walls' distance where the stress is maximum versus $\theta$. Continuous lines: fit with smoothing cubic splines.

The physical reason for this phenomenon is that at $\theta=1$ the liquid completely covers the atomically smooth surface and no crack-like defects occur at the interface; moreover the stored elastic energy at the interface is minimal. Thus the adhesion reaches a local maximum.

For the rough (self-affine fractal) substrate, the pull-off stress versus $\theta$ is shown in figure 3 . In this case the monolayer coverage corresponds to $\theta \approx 1.3$, i.e., the roughness increases the substrate surface area by about $30 \%$. For strong adsorbate interaction, the adhesion decreases with increasing liquid coverage up to $\theta \approx 0.2$ due to molecules trapped in the asperity contact regions, which effectively enhances the substrate surface roughness. For $\theta>0.2$ the pulloff force increases due to the formation of capillary bridges (see figure 5(c)). For weak adsorbate interaction, due to the strong wall-wall interaction and weak liquid-wall interaction, the liquid molecules are squeezed away from the asperity contact regions into the valleys (see figures 4(c), (d) and 7), which results in nearly constant pull-off force for $\theta<1$ (see curve 2 in figure 3). Due to the hydrophobic interaction between the liquid and the blocks, no capillary bridge forms, and as $\theta$ increases beyond 1 the pull-off force decreases towards zero.

For strong adsorbate interaction, the lateral corrugation of the molecule-substrate interaction potential is so high that liquid molecules are trapped in the asperity contact regions. 
(a)

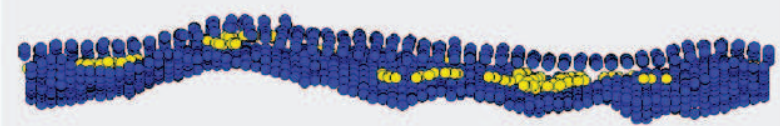

(b)

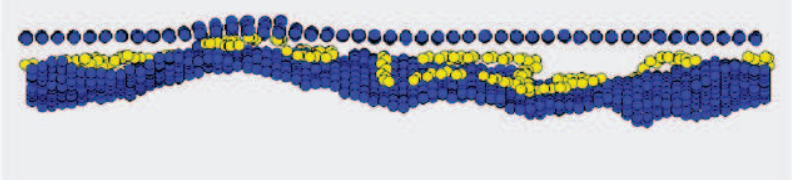

(c)

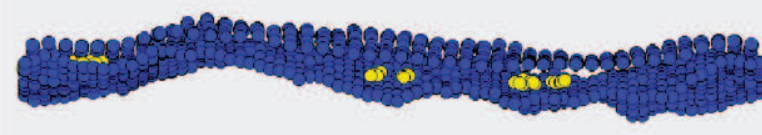

(d)

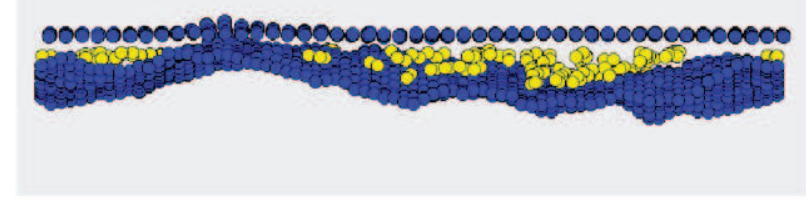

Figure 4. Side view of the contact for coverage $\theta=1 / 4$ on a rough substrate. The pictures show only the atoms in a $29 \AA$ thick slice including the highest asperity. (a) Strong adsorbate interaction; initial state. (b) Strong adsorbate interaction; maximum pull-off force. (c) Weak adsorbate interaction; initial state. (d) Weak adsorbate interaction; maximum pull-off force.

This is illustrated in figures 4(a) and (b) (side-view snapshots). The trapped molecules increase the elastic energy stored at the interface and reduce the effective wall-wall binding energy. Thus, for $\theta<0.67$ the pull-off stress for strong adsorbate interaction is smaller than that for weak interaction, as is shown in figure 3(a). For the rough substrate with strong adsorbate interaction, the pull-off stress reaches a maximum at $\theta \approx 1.3$ (see curve 1 in figure 3 ). During pull-off some liquid molecules are pulled out of the valleys (or cavities), and form nanosize capillary bridges in the asperity regions of the rough substrate (see figure 5), which results in an effective wall-wall interaction which is more long ranged than when the wall-liquid interaction is weak. For the weak adsorbate interaction case (see figure 6), the liquid does not wet the surface of the block (hydrophobic interaction) and no capillary bridges form, and for $\theta>1$ the pull-off stress is much smaller than that for strong adsorbate interaction.

The force-distance curves for the rough substrate with $\theta \approx 0.25$ and 1.3 are shown in figures 8 and 9 respectively, for both strong (a) and weak (b) adsorbate interaction. Note that for weak adsorbate interaction there is nearly no hysteresis in the stress. For strong adsorbate interaction large hysteresis is observed, and several abrupt changes in the pressure can be observed during squeezing, which correspond to the squeeze-out of some lubricant molecules from some asperity contact regions. 
(a)

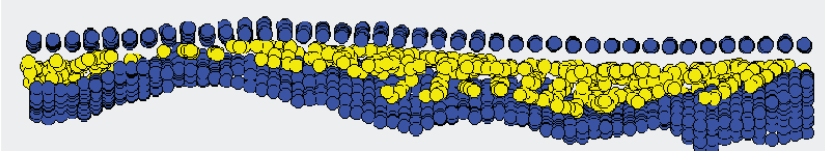

(b)

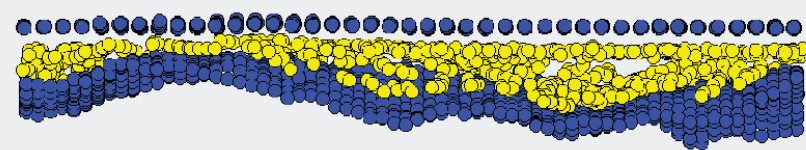

(c)

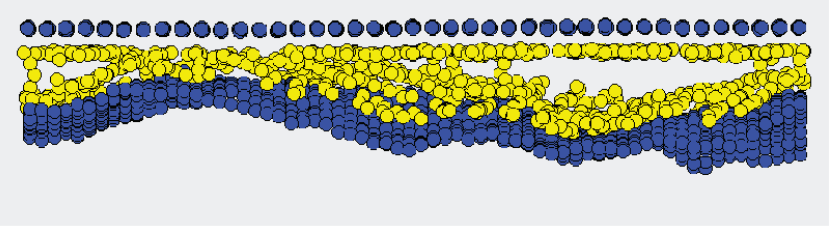

Figure 5. Side view of some atoms for the strong interacting adsorbate at coverage $\theta=1.3$ on a rough substrate, including the highest asperity. The system evolves in time from (a) to (b) and then to (c) during the pulling phase.

The breaking of the bonds between two macroscopic solids during pull-off is usually due to interfacial crack propagation, and the macroscopic pull-off force is determined by the energy (per unit of created surface area) $G(v)$ to propagate the crack at the velocity $v$, or, equivalently, the effective work of adhesion $\gamma_{\text {eff }}(v)$. For very small solid objects bond-breaking may occur more uniformly over the contact area and the standard picture described above no longer holds (see [25]). Thus, the results presented in this study may be directly relevant for adhesion involving small (micro- or nano-) sized solid objects, while the pull-off force for macroscopic objects may be more related to the work of adhesion during retraction, which can be obtained from pressure-distance plots such as those presented in figures 8 and 9 but not studied in detail in this paper.

Very recently Huber et al [13,14] have studied the influence of humidity on the nanoscale adhesion of individual gecko spatulae to smooth glass surfaces. The hierarchical gecko foot structure consists of fibre or hair-like structures, and each fibre ends with a $\sim 100 \mathrm{~nm}$ wide plate-like structure which is only a few nanometres thick at its thinnest place. The plates can easily bend to make contact even to very rough substrates [2]. Huber $e t$ al observed that the pull-off force on hydrophilic glass increased monotonically with increasing humidity. At the highest humidity the water film was $\sim 0.2 \mathrm{~nm}$ thick, which corresponds roughly to a water monolayer. This is consistent with our numerical results for rough surfaces and hydrophilic materials, which show that the pull-off force increases with increasing $\theta$ as $\theta$ increases from 0.2 to monolayer coverage (see figure 3 ). As the glass surface used in the experiments by Huber et al was very smooth, the dominant surface roughness may be that of the bottom surface of the plate-like structure of the spatula. When one spatula is completely submerged under water the pull-off force decreases by a factor $\sim 6$ as compared to humid conditions. 
(a)

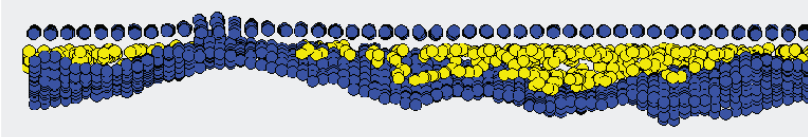

(b)

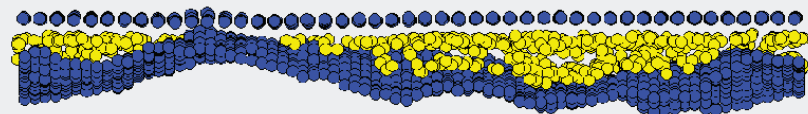

(c)

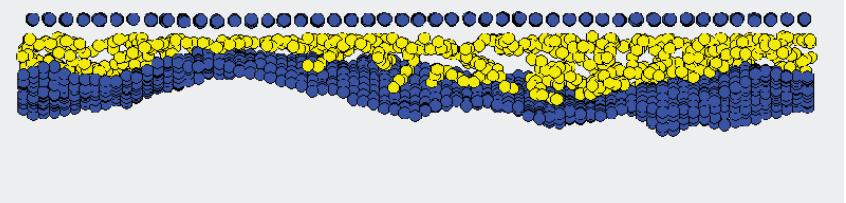

Figure 6. Side view of some atoms for the weak interacting adsorbate at coverage $\theta=1.3$ on a rough substrate, including the highest asperity. The system evolves in time from (a) to (b) and then to (c) during the pulling phase.

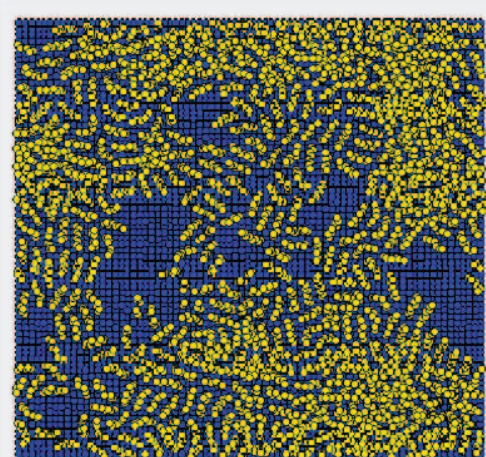

(a)

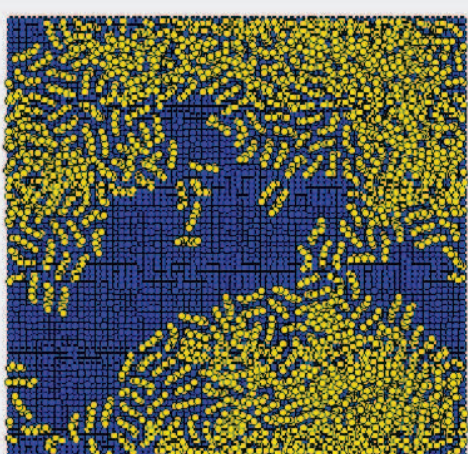

(b)

Figure 7. Top-view snapshots of the liquid on a rough substrate for $\theta \approx 1.3$, with (a) strong interaction and (b) weak interaction between liquid and walls. These images correspond to the side views shown in 5(a) and 6(a) respectively.

In conclusion, we have studied how molecularly thin liquid layers affect the adhesion between the solids both with smooth and rough surfaces. For strong interaction between the liquid and walls, the pull-off force exhibits a local maximum at monolayer coverage. For weak interaction, the pull-off force decreases continuously with increasing liquid coverage, especially when $\theta>1$ for rough substrate. It is clear that a fundamental understanding of the 

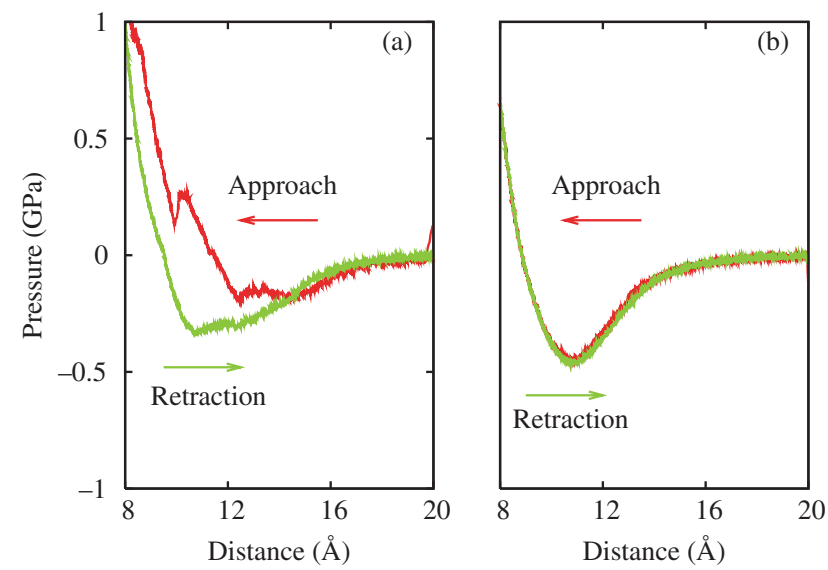

Figure 8. The average pressure as a function of the distance between the block and the rough substrate, with $\theta \approx 1 / 4 \mathrm{ML}$ of $\mathrm{C}_{8} \mathrm{H}_{18}$; (a) strong interaction; (b) weak interaction.
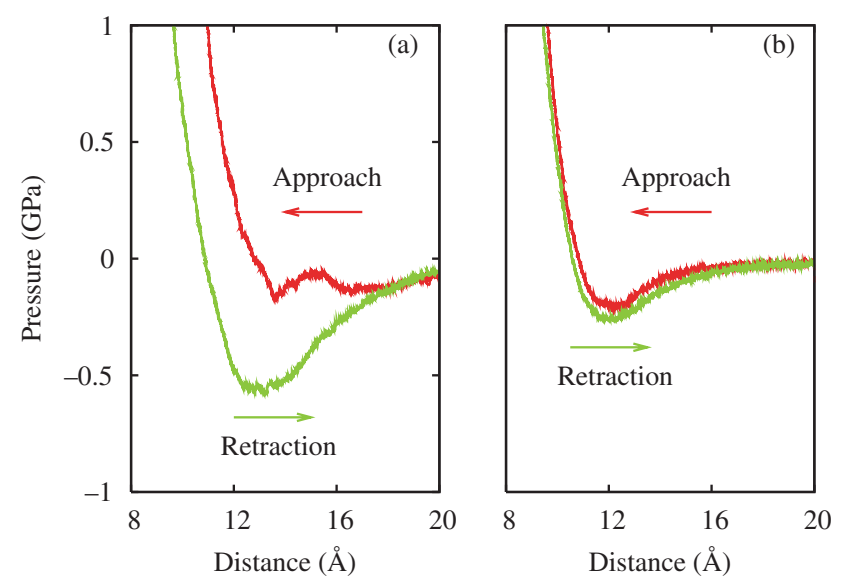

Figure 9. Average pressure as a function of the distance between the block and the rough substrate, with $\theta \approx 1.3 \mathrm{ML}$ of $\mathrm{C}_{8} \mathrm{H}_{18}$; (a) strong interaction; (b) weak interaction.

influence of liquid on adhesion at the microscale or nanoscale is central to a large number of biological and 'high-tech' engineering applications.

\section{Acknowledgments}

CY acknowledges the support from the International School for Advanced Studies (SISSA) and from the International Center of Theoretical Physics (ICTP) of Triest, Italy. The contribution of UT was partly sponsored by MIUR COFIN No. 2003028141-007, MIUR COFIN No. 2004028238-002, MIUR FIRB RBAU017S8 R004, and MIUR FIRB RBAU01LX5H.

\section{References}

[1] Scherge M and Gorb S 2000 Biological Micro- and Nano-tribology (Berlin: Springer)

[2] Persson B N J 2003 J. Chem. Phys. 1187614

Persson B N J and Gorb S 2003 J. Chem. Phys. 11911437 
[3] Persson B N J and Mugele F 2004 J. Phys.: Condens. Matter 16 R295

[4] See, e.g., Lawn B 1992 Fundamentals of Friction: Macroscopic and Microscopic Processes (NATO ASI Series, Series E: Applied Science vol 220) ed I L Singer and H M Pollock (Dordrecht: Kluwer) pp 137-65

[5] Persson B N J 2000 Sliding Friction: Physical Principles and Applications 2nd edn (Heidelberg: Springer)

[6] Israelachvili J 1992 Intermolecular and Surface Forces (London: Academic)

[7] Arzt E, Gorb S and Spolenak R 2003 Proc. Natl Acad. Sci. USA 10010603

[8] Heuberger M, Zach M and Spencer N D 2001 Science 292905

[9] http://adam.about.com/encyclopedia/19698.htm

[10] http://www.britannica.com/eb/article-9022138

[11] Lai-Fook S J 2004 Physiol. Rev. 84385

[12] Autumn K, Liang Y A, Hsieh S T, Zesch W, Chan W P, Kenny T W, Fearing R and Full R L 2000 Nature 405681

[13] Huber G, Martin H, Spolenak R, Mecke K, Jacobs K, Gorb S N and Arzt E 2005 Proc. Natl Acad. Sci. USA 10216293

[14] Huber G 2006 Nanoscale adhesion of individual Gecko spatula explored by atomic force microscopy Dissertation Universität Stuttgart, Bericht Nr. 183, Jan. 2006

[15] Zhao Y P, Wang L S and Yu T X 2003 J. Adhes. Sci. Technol. 17519

[16] Yang C Y and Zhao Y P 2004 J. Chem. Phys. 1205366

[17] Chandra S and Batur C 2006 Manipulation of capillary force by electrowetting for micromanipulation Nanotech. Technical Proceedings of the 2006 NSTI Nanotechnology vol 3 pp 578-81

[18] Samoilov V N, Sievebaek I M and Persson B N J 2004 J. Chem. Phys. 1219639

[19] Yang C, Tartaglino U and Persson B N J 2006 Phys. Rev. Lett. 97116103

[20] Yang C, Tartaglino U and Persson B N J 2006 Eur. Phys. J. E 1947

[21] Persson B N J, Albohr O, Tartaglino U, Volokitin A I and Tosatti E 2005 J. Phys.: Condens. Matter 17 R1

[22] Jorgensen W I, Madura J D and Swenson C J 1984 J. Am. Chem. Soc. 1066638

[23] Dysthe D K, Fuchs A H and Rousseau B 2000 J. Chem. Phys. 1127581

[24] Jensen P 1999 Rev. Mod. Phys. 711695

[25] Persson B N J 2003 Wear 254832 